\title{
2nd International Symposium on Materials Chemistry (ISMC-2008)
}

\section{Foreword}

The Special issue of this journal is based on the lectures delivered at the 2nd International Symposium on Materials Chemistry (ISMC-2008) held during 2-6 December 2008 at Bhabha Atomic Research Centre, Mumbai. The success of the first International Symposium on Materials Chemistry, organized during 4-8 December 2006, laid the foundation of ISMC series of symposia to be organized biennially. This series of symposia are fully supported by the Board of Research in Nuclear Sciences (BRNS) and the Society for Materials Chemistry.

There is ever increasing demand for advanced materials necessitating research and development activities in materials chemistry. The ISMC-2008 covered various aspects of materials chemistry with an emphasis on areas such as nuclear materials, nano materials, functional materials, superconductivity, materials for catalysis and chemical sensors. The ISMC-2008 was regarded as highly successful by international peers and reviewers. The wide range of topics covered in this Special issue reflect the trends emerging in materials chemistry.

We thank the Indian Academy of Sciences, Bangalore for agreeing to bring out this Special issue and the Editor, Journal of Chemical Sciences for support and encouragement. We are grateful to all the authors for their contributions and referees for their valuable help in evaluation of the manuscripts. Thanks are also due to the editorial staff of the journal for their unstinted support and cooperation.

15 January 2010

D DAS

Head, Chemistry Division

Bhabha Atomic Research Centre

Trombay, Mumbai 400085

e-mail: dasd@barc.gov.in

V K JAIN

Head, Synthetic and Pure Materials Section

Homi Bhabha National Institute

Trombay, Mumbai 400085

e-mail: jainvk@barc.gov.in

(Guest Editors) 